\title{
STUDY OF TUBERCULOSIS IN RHESUS MACAQUES OF NEPAL
}

\author{
S.P. Shrestha ${ }^{1 *}$, D. Shrestha ${ }^{1}$, M Prajapati ${ }^{1}$ and S.P Shrestha ${ }^{2}$ \\ ${ }^{1}$ Animal Health Research Division, NARC \\ ${ }^{2}$ Institute of Agriculture and Animal Science, TU \\ (*email:swoyamsps@yahoo.com)
}

\begin{abstract}
Tuberculosis is one of the prioritized zoonotic diseases in Nepal producing severe health hazard in humans and animals including both wildlife species and livestock species. It is also one of the most important bacterial diseases of nonhuman primates, especially macaques which is caused by Mycobacterium tuberculosis and M. bovis. A study was conducted from 2010 to 2012 in macaques of Nepal to find out the status of tuberculosis. Altogether 326 macaques were tested for tuberculosis by Intradermal Skin test on the eyelid and M. tuberculosis/M. bovis antibody testkit. Out of 326 macaques tested, 62 were found positive for the tuberculosis. So, the overall prevalence rate of tuberculosis in rhesus macaques was found to be $19.02 \%$. From the study it is concluded that not only humans, but the monkeys are also equally vulnerable to this disease condition and produce more severe health hazards, resulting even in death. These macaques should also be given priority concerning this as they may pose risk to other wild life species and humans. More researches should be directed towards exploring disease condition and status in these animals to protect other wildlife species and human contacting this disease.
\end{abstract}

Keywords: tuberculosis, zoonotic, macaques

\section{INTRODUCTION}

Tuberculosis (TB) is one of the prioritized zoonotic diseases in Nepal. It has been reported that it creates serious health hazard in nonhuman primates as well as to other animals and human as it causes economic and life losses in both (Min et al., 2013). It is one of the most important bacterial diseases of nonhuman primates, especially macaques which is caused by Mycobacterium tuberculosis \& M. bovis. Also, it has been reported that natural infection is not very common in primates i.e. they acquire illness primarily from humans and then spread rapidly within another monkey (Bennett et al., 1998). The main route of infection is via the respiratory tract by inhalation of fine particles containing the bacilli. Transmission may also occur by ingestion, through fight wounds, tattoo machines, needles, rectal thermometers, contact with fomites including contaminated thermo-fomites nets and gloves (Bennett et al., 1998 \& Lecu et al., 2013). So, other humans and animals with active disease represent the main reported sources of TB infection in captive primates. Retransmission of the infection to humans has been reported as well (Lecu et al., 2013).

Based on the incidence, the disease is common in Asian monkey species, especially those from India, less frequent in African species and rare in New World monkeys and prosimians (Lecu et al., 2013). More specifically, rhesus monkeys are more susceptible to M. tuberculosis than other experimental monkeys and apes (Min et al., 2013). Rhesus macaques develop an acute, progressive, 
and often fatal form of tuberculosis (Langermans et al., 2001). In nonhuman primates, it is well documented that the primary infection might not result in active TB and the latent infection might occur without any overt disease (Capuano et al., 2003; Gormus et al., 2004). Although animals with latent TB are not infectious, there is a significant risk of reactivation and the development of active tuberculosis (Lerche et al., 2008). So, macaques are considered to be disease reservoirs (Alfonso et al., 2004).

In macaques, TB classically involves the respiratory system; clinical signs may include coughing labored breathing and exercise intolerance (Bennett et al., 1998). However, the most common sign is cough. In some cases, it may remain subclinical for upto 6 months. The disease progresses slowly, taking up to one year to cause death in a rhesus. As TB progresses, monkeys may become fatigued, anorectic and experience chronic and marked emaciation. In general, intermittent coughing and chronic weight loss should always be regarded as possible indicators for the disease (Lecu et al., 2013). Hepatomegaly and splenomegaly in some cases may be detected by palpation. Hence, this study was conducted as there was an outbreak of T.B in the macaques breeding centre at Nepal Biotechnology Research Centre (NBRC), Lele, Lalitpur. The main objective of this study was to identify the T.B positive macaques and to isolate them from the breeding colony.

\section{MATERIALS AND METHODS}

A total of 326 macaques were collected from 2010-2012 A.D from various places of Nepal; Hetauda Bhutandevi temple (Hetauda), Melamchi (Sindhupalchok), Dhadingbeshi (Dhading), Parsa and Nepalgunj. These monkeys were collected from the places surrounding the human dwellings in case of Hetauda, Sindhupalchowk and Dhading. While from Parsa and Nepalgunj, they were collected from the woods. Thus, captivated monkeys were brought to Nepal Biomedical Research Center (NBRC), Lele-8, Lalitpur. For the diagnosis of tuberculosis in these macaques, 2 types of test procedures were used; Intradermal skin test on the eyelid (Delayed hypersensitivity test) and $\mathrm{M}$. tuberculosis/M. bovis rapid antibody test kit

\section{Intradermal skin test}

Macaques weighing above $1.5 \mathrm{~kg}$ were sedated with ketamine while baby macaques were properly restrained. Then, $0.1 \mathrm{ml}$ (irrespective of the body weight) of a solution containing $1250 \mathrm{IU}$ mammalian purified protein derivative (PPD), USA was intradermally injected into upper eyelid of eye (midway between the medial and lateral canthi) as shown in (Fig. 1 and 2). Subsequently, observations were made at 8, 24, 48 and 72 hours post inoculation. Positive and negative cases were identified based on the following changes in eyelid. (Table 1).

Table 1: Classification of tuberculin test

\begin{tabular}{lll}
\hline Test reaction & Changes in eyelid & Inference \\
\hline 0 & No reaction & Negative \\
1 & Bruising, no swelling & Negative \\
2 & Pronounced erythema of eyelid spread beyond the injection site, no swelling & Negative \\
3 & Slight swelling and drooping of eyelid. No redness noted & Indeterminate \\
4 & Marked drooping of eyelid due to swelling. Redness may be present or & Positive \\
& absent & \\
5 & Eyelid is swollen and closed. Redness may be present or absent & Positive \\
\hline
\end{tabular}




\section{M. tuberculosis/M. bovis rapid antibody test}

The antigen rapid bovine TB test kit is a chromatographic immunoassay for the qualitative detection of Mycobacterium bovis antibody in bovine whole blood, plasma or serum. The Antigen Rapid Bovine TB test kit shows two letters which are test (T) line and control $(\mathrm{C}$ line on the surface of device. The control line is a reference line which provides the test is performing properly. The control line has to appear at all times after the test has performed. If the antibodies against Mycobacterium bovis exist in sample, a purple test line would appear in the result window.

The highly selective recombinant Mycobacterium bovis antigens are used as a capture and detector in the assay. These antigens are capable of detecting Mycobacterium bovis antibodies in sample with high accuracy. For this, venous blood was collected from saphenous/ femoral vein, of which $30 \mu \mathrm{l}$ wasadded onto the center of the test well. After which 3 drops of diluents were added dropwise and reading was taken 20 minutes thereafter. Positive reading taken within after 20 minutes was shown as in (Fig. 3). Whereas, results read after 30 minutes were considered invalid.

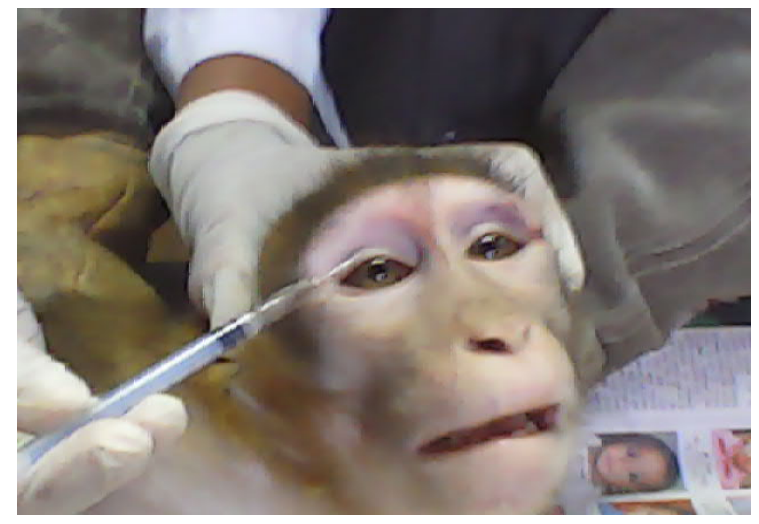

Fig. 1: Intradermal skin test on the eyelid

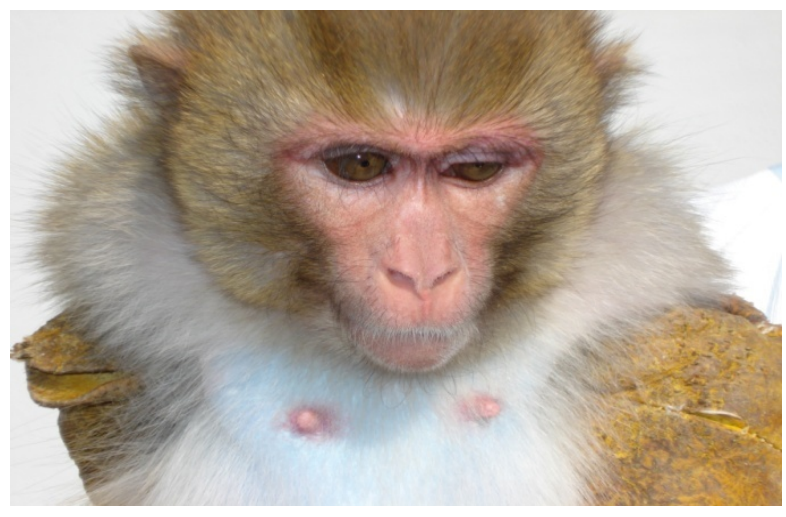

Fig.2: Positive reactor on intradermal test after 24 hour

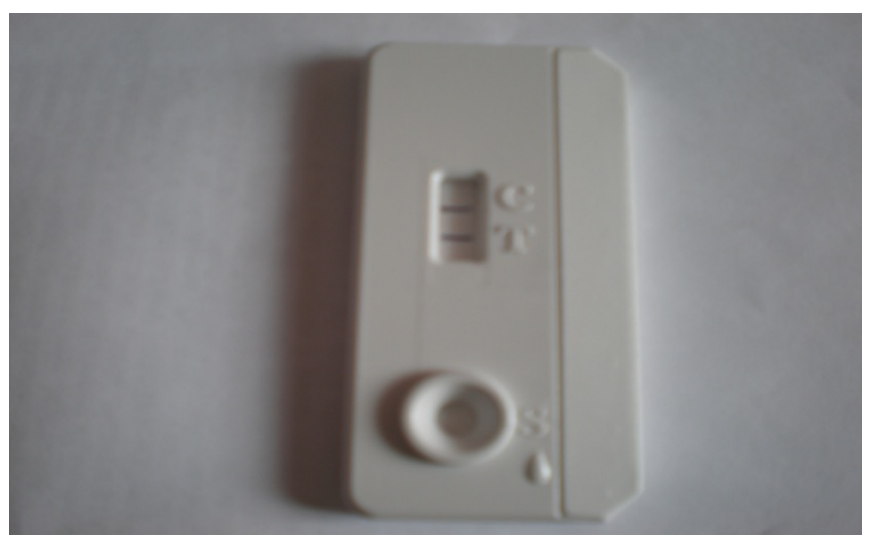

Fig. 3: Kit detecting positive TB specimen 


\section{RESULTS}

Out of the 326 macaques tested 62 were found positive both in PPD and antibody rapid test for the tuberculosis. All the 62 PPD positive reactors were subjected for rapid antibody test and all the positive reactors for PPD was also positive for rapid test. So, the overall prevalence of tuberculosis in rhesus macaques was found to be $19.02 \%$. At first the macaques were screened using the tuberculin test and the cases were classified as mentioned in (Table 1) above.

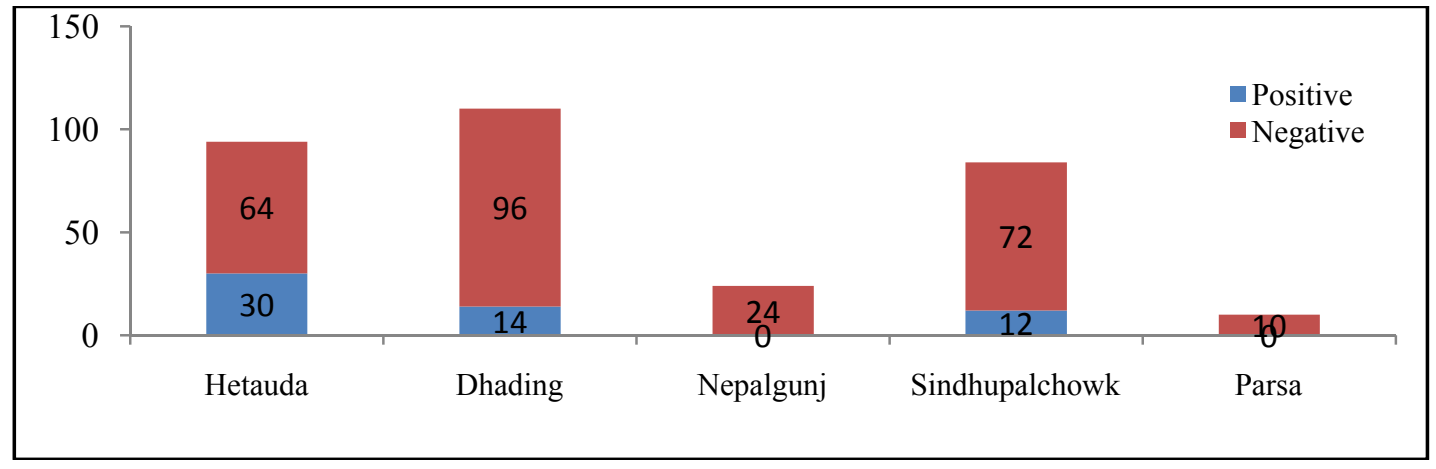

Fig.4: Proportion of positive cases in different locations

The cases positive to tuberculin test were further confirmed by the antibody test kit. The confirmed cases were euthanized, and post-mortem examination was done. In the postmortem examination, several multifocal pinpoints, miliary to nodular lesions were seen in various organs especially lungs, spleen, intestine, liver, trachea-bronchial and mesenteric lymph nodes, kidney as shown in (Fig. 4 to 7) below. The lymph nodes especially trachea-bronchial were enlarged with gray, soft, cheesy, somewhat gritty centers. Caseous tubercles were prominent in the lungs. Consolidation, airway exudation and gross bronchopneumonia were also seen in the lungs.

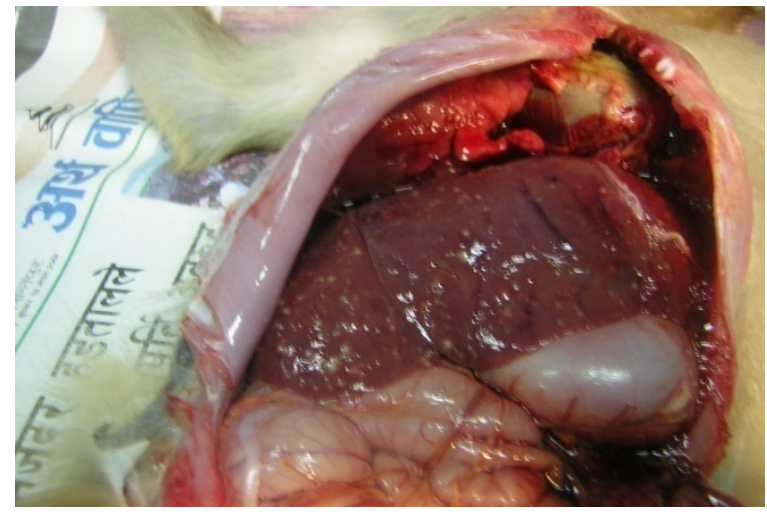

Fig.5: Viscera of Macaque died from TB

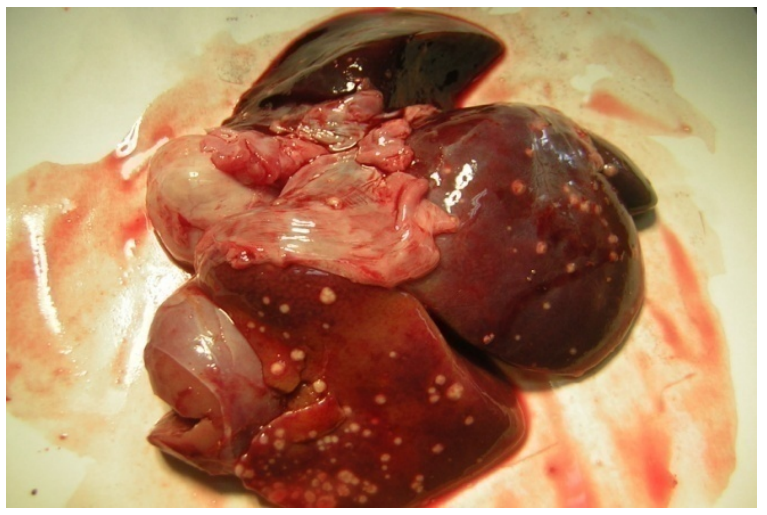

Fig.6: TB infected liver of macaque 


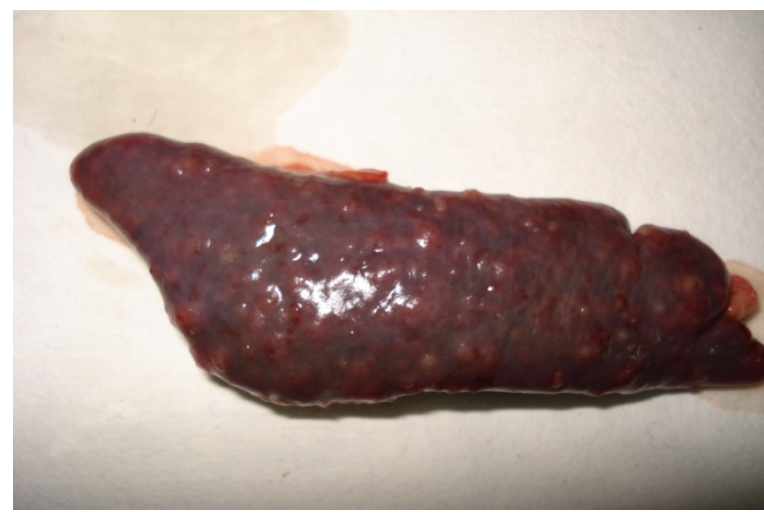

Fig.7: Spleen of a TB infected macaque

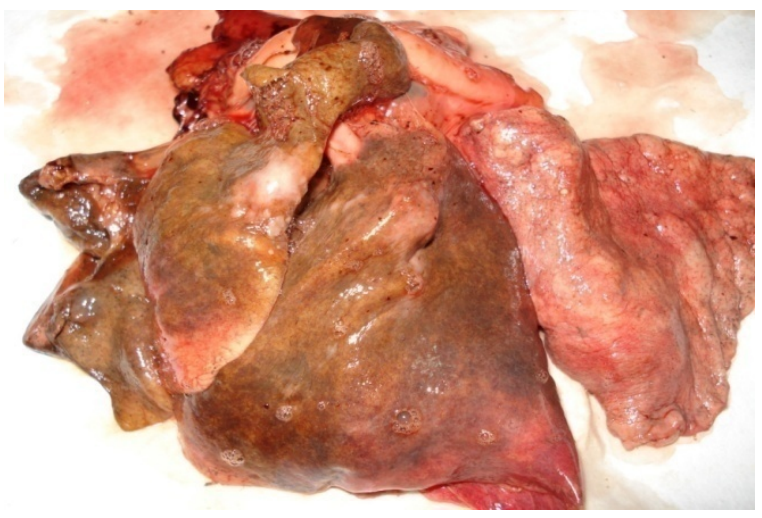

Fig. 8: Lungs of a TB infected macaque

\section{DISCUSSION}

Macaques are the lab animals used for the research based on mycobacterial tuberculosis mainly used for investigating tuberculosis pathogenesis and preclinical testing of drugs and vaccines owing to their anatomical and physiological similarities with human (Pena et al., 2015). However, natural infection is not seen in these nonhuman primates. It has been reported that the outbreak may have been through contact with an infected human, birds, contaminated water (Garcia et al., 2004). Similarly, the monkeys captured from Colombia where the human incidence of TB was high, were found to be infected with TB, suggesting that these animals could have acquired the infection through contact with tuberculous people (Alfonso et al., 2004). Our result also suggests the same finding as the macaques collected from woods from Parsa and Nepalgunj showed no tuberculosis at all. But the monkeys collected from Bhutandevi temple, Hetauda where monkeys roam around the temple, showed high infection rate. It suggests that these monkeys had high contact with humans (might be infected) visiting the temple. While the monkeys collected from Dhading and Sindhupalchowk were not always in close contact with humans as they returned to inhabitant after visiting the human premises for short interval. Also, during our research we noticed that there was rapid transmission of the pathogens among the healthy macaques from the sick ones. So, infection in captive macaques spread like wild fire.

Similarly, intradermal method and kit test were found to be equally efficient for the diagnosis of the disease condition. All the cases found positive by intradermal tests were found infective following kit test and post-mortem examination as well.

\section{CONCLUSION}

This study concludes that not only humans, but the monkeys are also equally vulnerable to this disease condition and produce more severe health hazards, resulting even in death. These macaques should also be given priority concerning Tuberculosis as they may pose risk to other wild life species and humans. Also, the control of human TB is impossible without control of TB in macaques- near human dwellings. Hence, more researches and initiatives should be directed towards exploring disease condition and status in these animals to protect other wildlife species and human contacting this disease. 


\section{ACKNOWLEDGEMENT}

The authors would like to thank all the staffs of Monkey Breeding Center, Lele for helping in collection of macaques and restraining them.

\section{REFERENCES}

Alfonso, R., Romero, R.E., Diaz, A., Calderon, M.N., Urdaneta, G., Arce, J. and Patarroyo, M.A. (2004). Isolation and identification of mycobacteria in New World primates maintained in captivity. Veterinary microbiology, 98(3): 285-295.

Bennett, B.T., Abee, C.R. and Henrickson, R. (1998). Nonhuman primates in biomedical research: Diseases. Academic Press.

Capuano, S.V., Croix, D.A., Pawar, S., Zinovik, A., Myers, A., Lin, P.L., \& Flynn, J.L. (2003). Experimental Mycobacterium tuberculosis infection of cynomolgus macaques closely resembles the various manifestations of human $\mathrm{M}$. tuberculosis infection. Infection and immunity, 71(10): 5831-5844.

Garcia, M.A., Bouley, D.M., Larson, M.J., Lifland, B., Moorhead, R., Simkins, M.D., ...and Otto, G. (2004). Outbreak of Mycobacterium bovis in a conditioned colony of rhesus (Macaca mulatta) and cynomolgus (Macaca fascicularis) macaques. Comparative medicine, 54(5): 578-584.

Gormus, B.J., Blanchard, J.L., Alvarez, X.H. and Didier, P.J. (2004). Evidence for a rhesus monkey model of asymptomatic tuberculosis. Journal of medical primatology, 33(3): 134-145.

Langermans, J.A., P. Andersen, D.van Soolingen, R.A. Vervenne, P.A. Frost, T. van der Laan, L.A. van Pinxteren, J. van den Hombergh, S. Kroon, I. Peekel, S. Florquin and A.W. Thomas. (2001). Divergent effect of bacillus Calmette-Guerin(BCG) vaccination on Mycobacterium tuberculosis infection in highlyrelated macaque species: implications for primate models in tuberculosisvaccine research. Proc. Natl. Acad. Sci. USA 98:11497-11502.

Lecu, A., Knauf, S., Mätz-Rensing, K. and Kaup, F.J. (2013). Tuberculosis in Nonhuman Primates, - an Overview of Diagnostic Tools.

Lerche, N.W., Yee, J.L., Capuano, S.V. and Flynn, J.L. (2008). New approaches to tuberculosis surveillance in nonhuman primates. ILAR journal,49(2): 170-178.

Min, F., Zhang, Y., Pan, J., Wang, J. and Yuan, W. (2013). Mycobacterium tuberculosis infection in rhesus monkeys (Macaca mulatta) and evaluation of ESAT-6 and CFP10 as immunodiagnostic antigens. Experimental Animals,62(4): 281-293.

Peña, J.C. and Ho, W.Z. (2015). Monkey models of tuberculosis: lessons learned. Infection and immunity, 83(3): 852-862. 